\title{
IWNLP: Inverse Wiktionary for Natural Language Processing
}

\author{
Matthias Liebeck and Stefan Conrad \\ Institute of Computer Science \\ Heinrich-Heine-University Düsseldorf \\ D-40225 Düsseldorf, Germany \\ \{liebeck, conrad\}@cs.uni-duesseldorf.de
}

\begin{abstract}
Nowadays, there are a lot of natural language processing pipelines that are based on training data created by a few experts. This paper examines how the proliferation of the internet and its collaborative application possibilities can be practically used for NLP. For that purpose, we examine how the German version of Wiktionary can be used for a lemmatization task. We introduce IWNLP, an opensource parser for Wiktionary, that reimplements several MediaWiki markup language templates for conjugated verbs and declined adjectives. The lemmatization task is evaluated on three German corpora on which we compare our results with existing software for lemmatization. With Wiktionary as a resource, we obtain a high accuracy for the lemmatization of nouns and can even improve on the results of existing software for the lemmatization of nouns.
\end{abstract}

\section{Introduction}

Wiktionary is an internet-based dictionary and thesaurus that lists words, inflected forms and relations (e.g. synonyms) between words. Just as Wikipedia, Wiktionary uses MediaWiki as a platform but focuses on word definitions and their meaning, rather than explaining each word in detail, as Wikipedia does. The dictionary contains articles, which can each list multiple entries for different languages and multiple parts of speech. For instance, the English word home has entries as a noun, verb, adjective and as an adverb.

Each article is rendered by the MediaWiki engine from a text-based input, which uses the MediaWiki syntax and relies heavily on the use of templates. The articles are editable by everyone,
Table 1: Declension of the German noun Turm (tower)

\begin{tabular}{|l|l|l|}
\hline Case & Singular & Plural \\
\hline \hline Nominative & der Turm & die Türme \\
\hline Genitive & $\begin{array}{l}\text { des Turmes } \\
\text { des Turms }\end{array}$ & der Türme \\
\hline Dative & $\begin{array}{l}\text { dem Turm } \\
\text { dem Turme }\end{array}$ & den Türmen \\
\hline Accusative & den Turm & die Türme \\
\hline
\end{tabular}

even by unregistered users. Although vandalism is possible, most of the vandalized entries are identified by other users who watch a list of the latest changes and subsequently revert these entries to previously correct versions. All text content is licensed under the Creative Commons License, which makes it attractive for academic use.

There are currently 111 localized versions of Wiktionary, which contain more than 1000 articles $^{1}$. A localized version can establish own rules via majority votes and public opinion. For example, the German version of Wiktionary ${ }^{2}$ currently enforces a 5-source-rule, which requires that each entry that is not listed in a common dictionary is documented by at least 5 different sources. The German version of Wiktionary has grown over the last years and currently contains almost 400000 articles $^{3}$. Each word is listed with its part-of-speech tag, among other information. If a word is inflectable (nouns, verbs, adjectives, pronouns and articles are inflectable in the German language), all inflected forms are also enumerated. Table 1 shows the declension of the noun Turm (tower). Wiktionary provides information that can be used as a resource for Natural Language Processing (NLP), for instance for part-of-speech tagging, for lemmatization and as a thesaurus.

\footnotetext{
${ }^{1}$ https://meta.wikimedia.org/wiki/Wiktionary

${ }^{2}$ https://de.wiktionary.org

${ }^{3}$ https://de.wiktionary.org/wiki/Wiktionary:Meilensteine
} 
The rest of the paper is structured as follows: Section 2 gives on overview of previous applications of Wiktionary for natural language processing purposes. Section 3 outlines the basic steps of parsing Wiktionary. The use of Wiktionary as a lemmatizer is evaluated in section 4 and compared with existing software for lemmatization. Finally, we conclude in chapter 5 and outline future work.

\section{Related Work}

The closest work to ours is JWKTL (Zesch et al., 2008). JWKTL is a Wiktionary parser that was originally developed for the English and the German version of Wiktionary, but it now also supports Russian. Our work differs from JWKTL, because we currently focus more on inflections in the German version than JWKTL. Therefore, we have a larger coverage of inflections, because we additionally reimplemented several templates from the namespace Flexion. Also, we have an improved handling of special syntactic cases, as compared to JWKTL.

Wiktionary has previously been used for several NLP tasks. The use of the German edition as a thesaurus has been investigated by Meyer and Gurevych (2010). The authors compared the semantic relations in Wiktionary with GermaNet (Hamp and Feldweg, 1997) and OpenThesaurus (Naber, 2005).

Smedt et al. (2014) developed a part-of-speech tagger based on entries in the Italian version of Wiktionary. They achieved an accuracy of 85,5\% with Wiktionary alone. By using morphological and contextual rules, they improve their tagging to an accuracy of $92,9 \%$. Li et al. (2012) also used Wiktionary to create a part-of-speech tagger, which is based on a hidden Markov model. Their evaluation of 9 different languages shows an average accuracy of $84,5 \%$, with English having the best result with an accuracy of $87,1 \%$.

\section{Parsing Wiktionary}

There are multiple ways to parse Wiktionary. It is possible to crawl all existing articles from the online servers. To reduce stress from the servers and to easily reproduce our parsing results, we parse the latest of the monthly XML dumps ${ }^{4}$ from Wiktionary. For this paper, we use the currently latest dump 20150407.

\footnotetext{
${ }^{4}$ http://dumps.wikimedia.org/dewiktionary/
}

We iterate over every article in the XML dump and parse articles which contain German word entries. These articles can be separated into two groups: the ones in the main namespace (without any preceding namespace, like 'namespace:') and the ones in the namespace Flexion. First, we describe how we parse the articles in the main namespace. An article can contain entries for multiple languages. Therefore, we divide its text content into language blocks (== heading $==$ ) and skip non-German language blocks. Afterward, we extract one or more entries (=== heading $===$ ) from each German language block. If an article lists more than one entry with the same name, its word forms will be different from each other. For instance, the German word Mutter $^{5}$, contains an entry for mother and for nut, which have different plural forms. We parse the part-of-speech tag for each entry. If a word is inflectable, we will also parse its inflections, which are listed in a keyvalue-pair template. Depending on the part-ofspeech tag, different templates are used in Wiktionary for which we use different parsers. We provide parsers for nouns, verbs, adjective and pronouns. The key-value-template for the adjective gelb (yellow) is displayed in Figure 1.

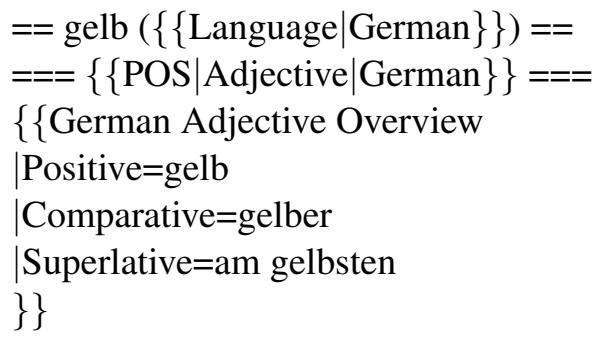

Figure 1: Adjective template for the word gelb (yellow), with keywords translated into English

At this point, we should point out that the inflections for verbs and adjectives in the main namespace are only a small portion of all possible inflections. For example, a verb in the main namespace only lists one inflection for the past tense (first person singular), while other possible past tense forms are not listed.

Fortunately, it is possible that a verb or an adjective has an additional article in the namespace Flexion, where all inflections are listed. However, the parsing of these inflections is more challenging, because the articles use complex templates.

\footnotetext{
${ }^{5}$ https://de.wiktionary.org/wiki/Mutter
} 
Although the parsing of the parameters for the templates remains the same, it is more difficult to retrieve the rendered output by the MediaWiki engine (and thus the inflections) from these templates, because it is very rare that inflections are listed as a key-value-pair. Instead, these templates require principal parts, which are combined with suffixes. The users of Wiktionary have created templates, that take care of special cases, for instance for a verb conjugation, where the suffix 'est' is added to a verb stem instead of 'st', if the last character of a verb stem is a ' $t$ '. Wiktionary uses a MediaWiki extension called ParserFunctions, which allows the use of control flows, like if-statements and switch statements. Special cases for the conjugation of verbs and the declension of adjectives are covered by a nested control flow. We have analyzed these templates and reimplemented the template of the adjectives and the most frequently used templates for verbs into IWNLP as $\mathrm{C \#}$ code. In total, Wiktionary currently contains 3705 verb conjugations in the Flexion namespace, which use several templates. We have limited our implementation to the three most used verb conjugation templates (weak inseparable $(51,4 \%)$, irregular $(27,2 \%)$, regular $(12,4 \%)$ ).

Altogether, we have extracted 74254 different words and 281457 different word forms. To reduce errors while parsing, we have written more than 150 unit tests to ensure that our parser operates as accurate as possible on various notations and special cases. During the development of IWNLP, we have manually corrected more than 200 erroneous Wiktionary articles, which contained wrong syntax or false content. To guarantee that we didn't worsen the quality of these articles, we've consulted experienced Wiktionary users before performing these changes.

Our parser and its output will be made available under an open-source license. ${ }^{6}$

\section{Lemmatization}

Wiktionary can be used as a resource for multiple NLP tasks. Currently, we are interested in using Wiktionary as a resource for a lemmatization task, where we want to determine a lemma for a given inflected form. For each lemma, Wiktionary lists multiple inflected forms. As outlined in section 3, we have parsed the inflected forms for each lemma. For our lemmatization task, we inverse

\footnotetext{
${ }^{6}$ http://www.iwnlp.com
}

this mapping to retrieve a list of possible lemmas for a given inflection, hence our project name IWNLP. For example, we use the information presented in Table 1 to retrieve Türme $\mapsto$ Turm. For each lemma $l$ in Wiktionary, we have also added a mapping $l \mapsto l$. Our mapping will also be available via download.

It is possible, that an inflected form maps to more than one lemma. For instance, the word Kohle maps to Kohle (coal) and Kohl (cabbage). In total, our mapping contains 2035 words, which map to more than one lemma.

With this paper, we want to evaluate how good Wiktionary performs in a lemmatization task. Additionally, we want to validate our assumption, that by first looking up word forms and their lemmas in Wiktionary, we should be able to improve the performance of existing software for lemmatization.

Therefore, we evaluate IWNLP and existing software on three German corpora, which list words and their lemmas: TIGER Corpus (Brants et al., 2004), Hamburg Dependency Treebank (HDT) (Foth et al., 2014) and TüBa-D/Z (Telljohann et al., 2012) release 9.1. The TIGER Corpus consists of 50472 sentences from the German newspaper Frankfurter Rundschau. The Hamburg Dependency Treebank (part A) contains 101981 sentences from the German IT news site Heise online. The TüBa-D/Z corpus comprises of 85358 sentences from the newspaper die tageszeitung (taz). Each word in these corpora is listed with its part-of-speech tag from the STTS tagset (Schiller et al., 1999). We evaluate the lemmatization for nouns (POS tag $N N$ ), verbs (POS tags $V^{*}$ ) and adjectives (POS tags $A D J A$ and $A D J D$ ). Due to the low amount of different articles and pronouns in the German language, we ignore them in our evaluation.

In our experiments, we look up the nouns, verbs and adjectives from each corpus in IWNLP. If we map a word form to more than one lemma in IWNLP, we treat this case as if there would be no entry for this particular word form in IWNLP. The same policy is applied in all of our experiments. We preserve case sensitivity, which worsens our results slightly. In a modification, that we name keep, we assume that a word $w$ will be its own lemma, if $w$ does not have an entry in the mapping. IWNLP is compared with a mapping ${ }^{7}$ ex-

\footnotetext{
${ }^{7}$ http://www.danielnaber.de/morphologie/index_en.html
} 


\begin{tabular}{|l|c|c|c|c|c|c|c|c|c|}
\hline \multirow{2}{*}{ Method } & \multicolumn{3}{|c|}{ TIGER Corpus } & \multicolumn{3}{c|}{ TüBa-D/Z } & \multicolumn{3}{c|}{ HDT } \\
\cline { 2 - 10 } & Noun & Verb & Adj & Noun & Verb & Adj & Noun & Verb & Adj \\
\hline \hline IWNLP & 0,734 & 0,837 & 0,633 & 0,720 & 0,809 & 0,567 & 0,607 & 0,864 & 0,613 \\
IWNLP + keep & $\mathbf{0 , 8 9 4}$ & 0,854 & 0,692 & 0,897 & 0,827 & 0,650 & 0,647 & 0,882 & 0,699 \\
Morphy & 0,196 & 0,713 & 0,531 & 0,181 & 0,671 & 0,490 & 0,163 & 0,675 & 0,475 \\
Morphy + keep & 0,857 & 0,962 & 0,763 & 0,860 & 0,916 & 0,744 & 0,619 & 0,963 & 0,735 \\
\hline Mate Tools & - & - & - & 0,926 & 0,927 & $\mathbf{0 , 8 5 2}$ & 0,639 & 0,971 & 0,712 \\
TreeTagger & 0,860 & $\mathbf{0 , 9 7 4}$ & 0,867 & 0,848 & 0,930 & 0,832 & 0,611 & $\mathbf{0 , 9 7 7}$ & 0,687 \\
\hline IWNLP + Mate Tools & - & - & - & $\mathbf{0 , 9 4 3}$ & 0,929 & 0,841 & $\mathbf{0 , 6 5 3}$ & 0,976 & $\mathbf{0 , 7 5 1}$ \\
Morphy + Mate Tools & - & - & - & 0,918 & $\mathbf{0 , 9 3 2}$ & 0,837 & 0,627 & 0,974 & 0,744 \\
IWNLP + TreeTagger & 0,888 & 0,969 & $\mathbf{0 , 8 6 9}$ & 0,879 & 0,927 & 0,795 & 0,641 & 0,973 & 0,724 \\
Morphy + TreeTagger & 0,859 & 0,970 & 0,810 & 0,843 & 0,926 & 0,787 & 0,602 & 0,968 & 0,713 \\
\hline
\end{tabular}

Table 2: Lemmatization accuracy for nouns, verbs and adjectives in all three corpora

tracted from Morphy (Lezius et al., 1998), a tool for morphological analysis.

For our comparison with existing software, that can be used for lemmatization, we have chosen Mate Tools (Björkelund et al., 2010) and TreeTagger (Schmid, 1994), which both accept tokenbased input.

The results of our experiments are shown in Table 2 . In a direct comparison between IWNLP and Morphy, IWNLP outperforms Morphy in the basic variant in all POS tags across all corpora. With the modification keep, the results of IWNLP and Morphy improve. IWNLP + keep is still superior for nouns, but Morphy + keep achieves better results for verbs and adjectives. The results from Mate Tools on the TIGER Corpus are excluded from Table 2 because Mate Tools was trained on the TIGER Corpus and, therefore, cannot be evaluated on it. The direct comparison of Mate Tools and TreeTagger shows that Mate Tools achieves an accuracy that is at least $2 \%$ better in four of the six cases. In the other two cases, TreeTagger only performs slightly better.

For the lemmatization of nouns, IWNLP is able to improve on the results of Mate Tools and TreeTagger across all three corpora. In total, IWNLP enhances the results of Mate Tools in five of the six test cases. Surprisingly, the additional lookup of word forms in IWNLP and Morphy can impair the accuracy for verbs and adjectives. In our future work, we will systematically analyze which words are responsible for worsening the results, correct their Wiktionary articles and improve our lookup in IWNLP.

The overall bad performance for the lemmatization of nouns in the HDT corpus can be explained by the gold lemmas for compound nouns, which are often defined as the last word in the compound noun. For instance, HDT defines that Freiheit (freedom) is the gold lemma for Meinungsfreiheit (freedom of speech).

\section{Conclusion}

We have presented IWNLP, a parser for the German version of Wiktionary. The current focus of the parser lies in the extraction of inflected forms. They have been used to construct a mapping from inflected forms to lemmas, which can be utilized in a lemmatization task. We evaluated our IWNLP lemmatizer on three German corpora. The results for the lemmatization of nouns show that IWNLP outperforms existing software on the TIGER Corpus and can improve their results on the TüBa-D/Z and the HDT corpora. However, we have also discovered that we still need to improve IWNLP to get better results for the lemmatization of verbs and adjectives. We will try to resolve the correct lemma for an inflected form if multiple lemmas are possible.

Additionally, IWNLP will be extended to parse hyponyms and hypernyms for nouns. We plan to compare the use of Wiktionary as thesaurus with GermaNet (Hamp and Feldweg, 1997).

We expect that the presented results for the lemmatization task will improve with every new monthly dump if Wiktionary continues to grow and improve through a community effort.

\section{Acknowledgments}

This work is part of the graduate school NRW Fortschrittskolleg Online-Partizipation. We thank 
the Wiktionary user Yoursmile for his help.

\section{References}

Anders Björkelund, Bernd Bohnet, Love Hafdell, and Pierre Nugues. 2010. A High-Performance Syntactic and Semantic Dependency Parser. In Proceedings of the 23rd International Conference on Computational Linguistics: Demonstrations, COLING '10, pages 33-36. Association for Computational Linguistics.

Sabine Brants, Stefanie Dipper, Peter Eisenberg, Silvia Hansen-Schirra, Esther König, Wolfgang Lezius, Christian Rohrer, George Smith, and Hans Uszkoreit. 2004. TIGER: Linguistic Interpretation of a German Corpus. Research on Language and Computation, 2(4):597-620.

Kilian A. Foth, Arne Köhn, Niels Beuck, and Wolfgang Menzel. 2014. Because Size Does Matter: The Hamburg Dependency Treebank. In Proceedings of the Ninth International Conference on Language Resources and Evaluation (LREC-2014), pages 23262333.

Birgit Hamp and Helmut Feldweg. 1997. GermaNet a Lexical-Semantic Net for German. In Proceedings of ACL workshop Automatic Information Extraction and Building of Lexical Semantic Resources for NLP Applications, pages 9-15.

Wolfgang Lezius, Reinhard Rapp, and Manfred Wettler. 1998. A Freely Available Morphological Analyzer, Disambiguator and Context Sensitive Lemmatizer for German. In Proceedings of the 17th International Conference on Computational Linguistics Volume 2, COLING '98, pages 743-748. Association for Computational Linguistics.

Shen Li, João V. Graça, and Ben Taskar. 2012. Wiki-ly Supervised Part-of-speech Tagging. In Proceedings of the 2012 Joint Conference on Empirical Methods in Natural Language Processing and Computational Natural Language Learning, EMNLP-CoNLL '12, pages 1389-1398. Association for Computational Linguistics.

Christian M. Meyer and Iryna Gurevych. 2010. Worth Its Weight in Gold or Yet Another Resource - A Comparative Study of Wiktionary, OpenThesaurus and GermaNet. In Computational Linguistics and Intelligent Text Processing, 11th International Conference, CICLing 2010, volume 6008 of Lecture Notes in Computer Science, pages 38-49. Springer.

Daniel Naber. 2005. OpenThesaurus: ein offenes deutsches Wortnetz. In Sprachtechnologie, mobile Kommunikation und linguistische Ressourcen: Beiträge zur GLDV-Tagung 2005 in Bonn, pages 422-433. Peter-Lang-Verlag.

Anne Schiller, Simone Teufel, Christine Stöckert, and Christine Thielen. 1999. Guidelines für das Tagging deutscher Textcorpora mit STTS (kleines und großes Tagset). Technical report, Universität Stuttgart, Universität Tübingen.

Helmut Schmid. 1994. Probabilistic Part-of-Speech Tagging Using Decision Trees. In Proceedings of the International Conference on New Methods in Language Processing.

Tom De Smedt, Fabio Marfia, Matteo Matteucci, and Walter Daelemans. 2014. Using Wiktionary to Build an Italian Part-of-Speech Tagger. In Natural Language Processing and Information Systems - 19th International Conference on Applications of Natural Language to Information Systems, NLDB 2014, volume 8455 of Lecture Notes in Computer Science, pages 1-8. Springer.

Heike Telljohann, Erhard W. Hinrichs, Sandra Kübler, Heike Zinsmeister, and Kathrin Beck. 2012. Stylebook for the Tübingen Treebank of Written German (TüBa-D/Z). Technical report, University of Tübingen.

Torsten Zesch, Christof Müller, and Iryna Gurevych. 2008. Extracting Lexical Semantic Knowledge from Wikipedia and Wiktionary. In Proceedings of the Sixth International Conference on Language Resources and Evaluation (LREC'08). European Language Resources Association. 